\title{
Implementation of a Probabilistic Structural Health Monitoring Method on a Highway Bridge
}

\author{
Adam Scianna, Zhaoshuo Jiang, Richard Christenson, and John DeWolf \\ Department of Civil and Environmental Engineering, University of Connecticut, Storrs, Connecticut 06269-2037, USA \\ Correspondence should be addressed to Richard Christenson, rchriste@engr.uconn.edu
}

Received 30 June 2011; Revised 25 October 2011; Accepted 29 December 2011

Academic Editor: Piervincenzo Rizzo

Copyright () 2012 Adam Scianna et al. This is an open access article distributed under the Creative Commons Attribution License, which permits unrestricted use, distribution, and reproduction in any medium, provided the original work is properly cited.

This paper describes the application of a probabilistic structural health monitoring (SHM) method to detect global damage in a highway bridge in Connecticut. The proposed method accounts for the variability associated with environmental and operational conditions. The bridge is a curved three-span steel dual-box girder bridge located in Hartford, Connecticut. The bridge, monitored since Fall 2001, experienced a period of settling in the Winter of 2002-2003. While this change was not associated with structural damage, it was observed in a permanent rotation of the bridge superstructure. Three damage measures are identified in this study: the value of fundamental natural frequency determined from peak picking of autospectral density functions of the bridge acceleration measurements; the magnitude of the peak acceleration measured during a truck crossing; the magnitude of the tilt measured at 10-minute intervals. These damage measures, including thermal effects, are shown to be random variables and associated $P$ values are calculated to determine if the current probability distributions are the same as the distributions of the baseline bridge data from 2001. Historical data measured during the settling of the bridge is used to verify the performance of the bridge, and the field implementation of the proposed method is described.

\section{Introduction}

Structural health monitoring (SHM) is a general term used in many engineering disciplines that describes a process to determine the integrity of a structure. There have been numerous techniques developed to ascertain the integrity of a structure including methods of visualization, nondestructive evaluation (NDE) as well as indentifying changes in vibration characteristics. All of these methods have a common goal to detect, locate, and quantify varying degrees of deterioration and damage within a structure.

Visual inspection and NDE are common methods of health monitoring. Visual inspection, however, is neither objective nor reliable, containing great uncertainty in identifying the existence, location, and degree of damage [1-3]. Additionally, visual inspection supplies information only at the time of inspection. Realistic SHM should supply information on a continuous basis if it is to be of real use. New technologies for the NDE of civil structures include ultrasonic testing, penetrant testing, visual testing (different than visual inspec- tion), magnetic particle testing, radiographic testing, acoustic emission, and eddy current testing $[4,5]$. These technologies determine local damage and typically require the general region of damage on the structure to be first identified. For many structures, locating the damage prior to inspection is difficult.

Using vibration measurements is a less subjective method for structural health monitoring that can allow for global as well as local evaluation. As such, vibration-based techniques are receiving much recent attention [6-14]. Employing methods that rely on ambient vibration or operational loads are challenging as these excitations tend to excite the lower frequency global modes that can be insensitive to local damage [15]. Furthermore, environmental and operational variability of civil structures can affect the natural frequencies and mode shapes, rendering SHM methods that rely on changes in these parameters ineffective except in the presence of extreme damage [16]. Despite these challenges, using vibration measurements for SHM has continued to receive the attention of researchers and is the focus of this study. 
Bridge health monitoring (BHM), a type of SHM applied specifically to bridges, has been applied in Connecticut since 1984, when the University of Connecticut and the Connecticut Department of Transportation began evaluating the structural conditions of bridges in the state [17-19]. Since that time there have been various temporary sensor installations that have helped determine in-service behavior and justify rehabilitation and repair plans [19]. The portable monitoring system applications have provided benefits in the form of fewer or no repairs totaling over $\$ 2.5 \mathrm{M}$ and have identified necessary repairs increasing safety. A long-term continuous bridge monitoring program has been in place since 1997, when permanent monitoring systems were installed on a variety of bridge types across Connecticut. The objective of the continuous monitoring is to identify global changes in a bridge's behavior over multiple years [17]. The bridge examined in this study is part of the long-term continuous BHM program in Connecticut.

Prior work conducted at the University of Connecticut studied the effects of damage on the dynamic properties of a bridge and the effects of temperature variation on these measurements. This previous work indicates that monitoring the bridge's modal information as well as peak values can provide a global measure of the bridge's structural integrity and identify major changes in the structural integrity [20]. In addition, tiltmeters are employed to measure the general orientation of the bridge as a measure of its health in terms of structural changes. The effect of temperature on modal information and tilt measurements have been identified and quantified in Liu and DeWolf [21] and Olund [22].

While the tools for statistical analysis are numerous and well established, there has been limited research applying these methods to BHM [12]. A statistical pattern recognition paradigm was proposed by Rytter [23]. More recently, Zhang [24] showed that by selecting a damage feature, characterized as a random variable with a normal distribution, a probability of damage could be determined by comparing a feature of an unknown condition to that of a known condition. Olund and DeWolf [20] established the basis for BHM in Connecticut showing a statistical approach that can be very robust with respect to environmental and operational variability.

This paper extends the statistical BHM approach of Olund and DeWolf [20] to examine the vibration characteristics and tilt of an actual highway bridge in Connecticut verifying the proposed approach using historical data collected over a period that included a permanent rotation in the bridge deck. This paper goes on to describe the implementation of a fully automated BHM system on an in-service highway bridge in Connecticut.

\section{Bridge and Monitoring System Description}

The bridge selected for this study, part of the long-term continuous BHM program in Connecticut, is referred to as the Hartford Flyover Bridge. This bridge connects I-84 east to I91 north in Hartford, CT, USA. Figure 1 shows an aerial view of the Hartford Flyover Bridge.

The structure is comprised of two steel box girders with a composite concrete deck. The bridge in its entirety is nine

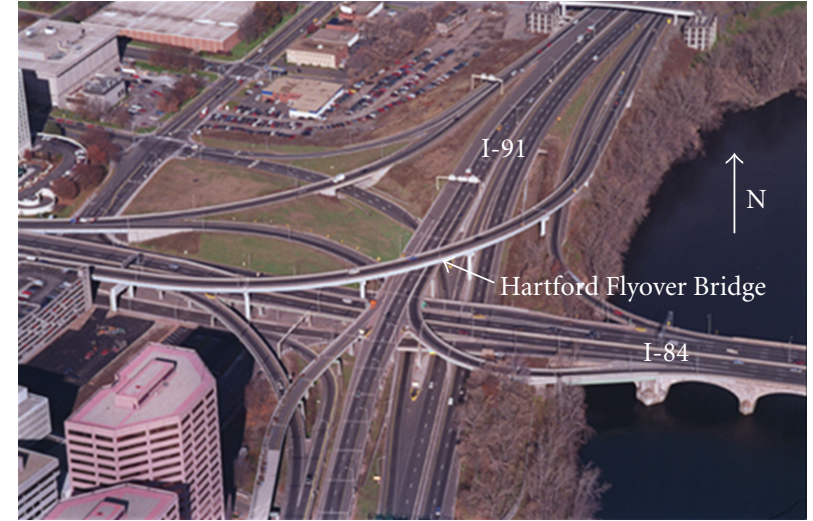

Figure 1: Aerial view of the Hartford Flyover Bridge.

spans consisting of three sets of three continuous spans which are simply supported. Previous studies have identified the fundamental natural frequency of the continuous bridge span being monitored being equal to approximately $1.52 \mathrm{~Hz}$ [22]. The monitoring system's 22 sensors include 8 accelerometers, 8 temperature transducers and 6 tiltmeters. Figure 2 shows the location of the various sensors on the bridge. All sensors are located in or on the box girder of the middle continuous three span segment. This is the portion of the bridge with the longer columns. The sensors were distributed to capture the behavior of this segment of the bridge in order to determine the cause of cracking in the supporting columns observed during the biannual visual inspection. The tiltmeters and accelerometers are located along the length of the bridge while all the temperature transducers are located at the midspan cross-section of span 4 .

The accelerometers are PCB Piezotronics Model 393C quartz accelerometers with a measurement range of $\pm 2.5 \mathrm{~g}$ and a frequency range of $0.025-800 \mathrm{~Hz}$. Six of the accelerometers measure vertical accelerations and two measure horizontal accelerations. These are labeled as $\mathrm{AV} \#$ and $\mathrm{AH}$, respectively, in Figure 2. The acceleration measurements are dynamic measurements collected at a sampling rate of $90.91 \mathrm{~Hz}$ for a duration of 30 seconds when a truck travels over the bridge and triggers sensor AV2 to exceed the prescribed $0.0095 \mathrm{~g}$ threshold. The data acquisition system used to collect data during 2002-2003 period employs a 2-pole analog low-pass filter with a cut-off frequency of $2 \mathrm{~Hz}$. As such, the monitoring system is able to capture the frequency content of only the fundamental mode without distortion. The current upgraded system provides a bandwidth up to $500 \mathrm{~Hz}$. A comparison of the autospectral density function measured from AV1 measured with the original and upgraded systems in shown in Figure 3. Both measurements show the fundamental natural frequency at $1.52 \mathrm{~Hz}$ as well as higher frequencies above $2 \mathrm{~Hz}$. It is observed that higher modes are excited by the crossing truck traffic and higher modes can be used in current studies.

Tiltmeters 1, 2, 4, 5, and 6, applied geomechanics model 801-S \pm 3 degrees, are located at the piers while tiltmeter 3 is located at midspan of Span 4, collocated with the array of 


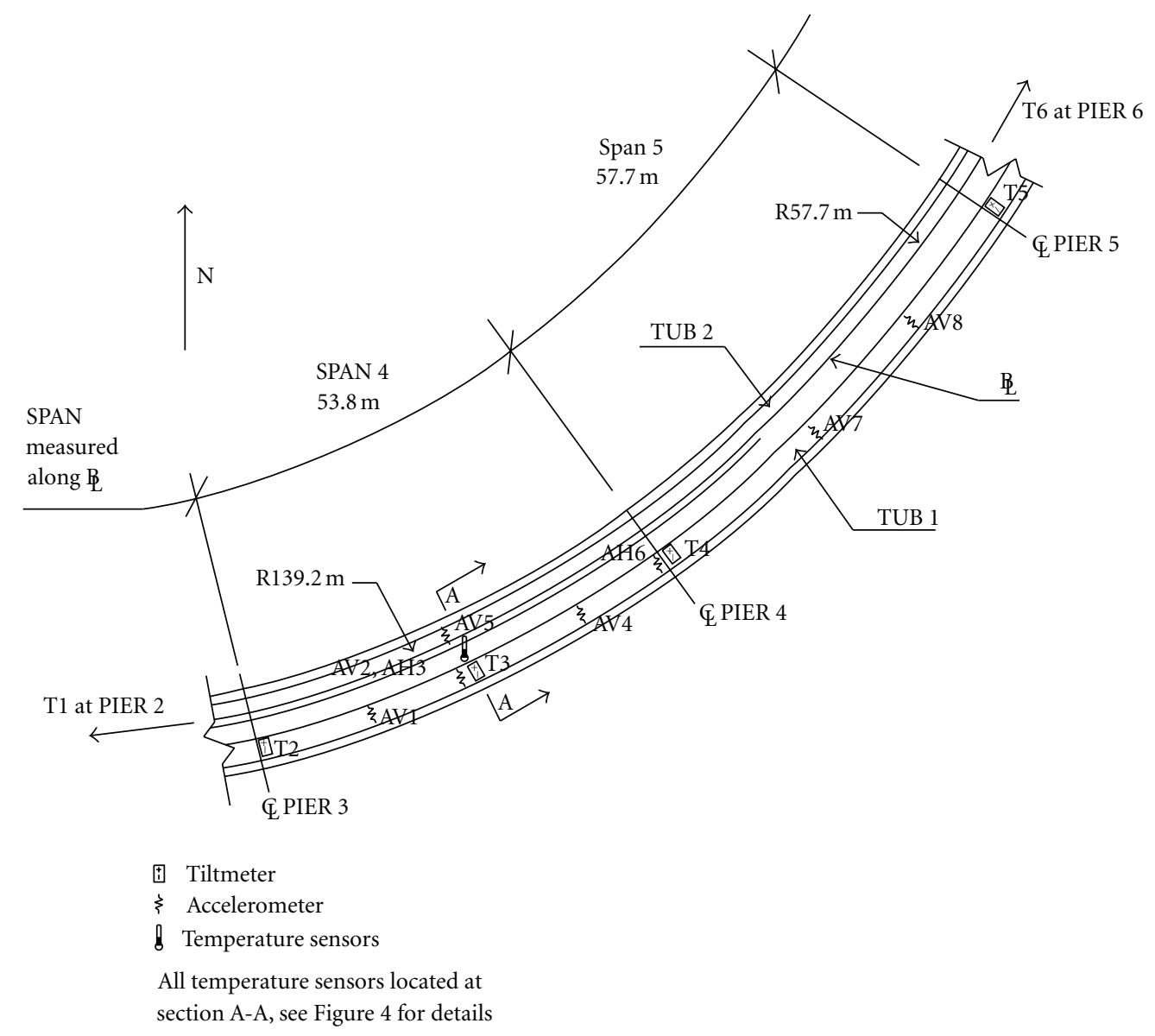

Figure 2: Sensor locations for the Hartford Flyover Bridge.

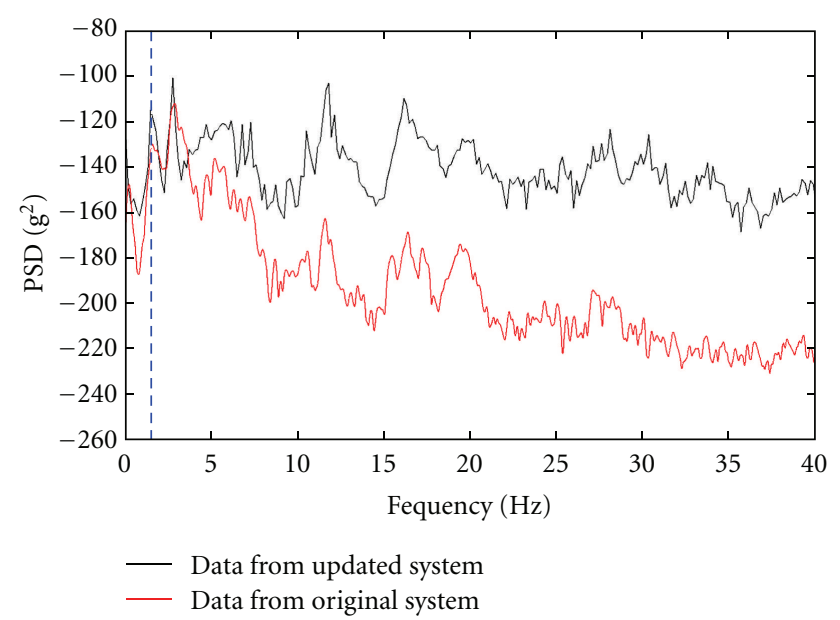

FIGURE 3: Autospectral density function of AV1 (vertical dashed line at fundamental natural frequency, $1.52 \mathrm{~Hz}$ ).

temperature sensors. Tilt measurements are collected at regular ten-minute intervals.

The temperature transducers are RdF model 29258 RTD capsules for measuring ambient and inside deck temperatures. RdF surface flange mount model 22802 are used for measuring surface temperatures. The transducers provide temperature measurements with a $0.041^{\circ} \mathrm{C}\left(0.074^{\circ} \mathrm{F}\right)$ resolution. Of the eight temperature transducers, two measure the concrete deck temperature, four measure the temperature in the steel tub corners, and the other two record ambient temperature both inside and outside the tub. These transducers were originally placed in this configuration to study the effects of a temperature differential on tilt in the cross-section of a steel girder bridge with a composite concrete deck [25]. The temperature detector locations are identified in Figure 4. Temperature measurements are also collected at regular ten minute intervals corresponding to the tilt measurements. The eight temperature measurements are closely correlated and as such only the measurement of the first temperature sensor, R1, is employed in this study.

\section{Probabilistic Structural Health Monitoring Method}

To address the challenges of environmental and operational variability, including unknown traffic excitation, inherent variability of the excitation, measurement error, and uncertainty in the structure itself, a probabilistic framework is adopted. In this probabilistic method damage measures (DMs) corresponding to the structural health of the bridge are determined from the structure's dynamic response and 


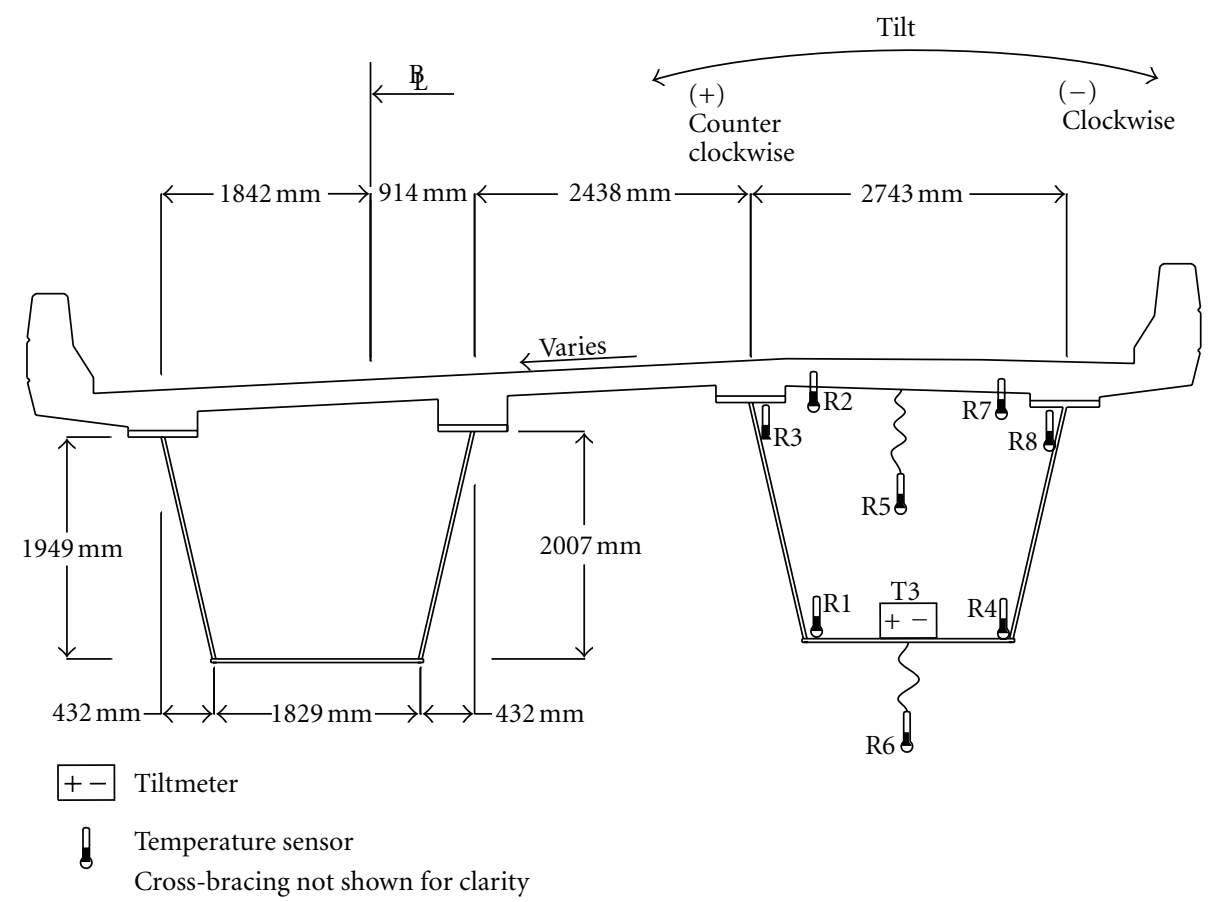

FIgURE 4: Cross-section of the Hartford Flyover Bridge with temperature sensor layout.

rotation of the bridge deck. The basis for this method is that a baseline damage measure $\left(D_{B}\right)$ can first be determined. At subsequent times, the current damage measure $\left(D_{C}\right)$ can be determined and compared to the baseline case. For this study the DMs used are: (i) the estimated fundamental natural frequency of the bridge, calculated as the first peak of the power spectral density function (PSD) of the acceleration; (ii) the peak acceleration resulting from a truck crossing over the bridge; (iii) the 10-minute tilt measurements of the bridge. Although almost any damage measure proposed in the literature can be used, these three benchmark parameters were chosen because of prior experience in BHM in Connecticut [18].

The DMs, calculated from measured responses for varying traffic loading, are observed from the approximate straight lines in the normal probability plots to be random variables with a Gaussian distribution. As random variables, not just one realization of the baseline DM is calculated, but a set of $n$ baseline DMs are determined. The mean and variance of an original set of data for the baseline structure is determined so that the probability density function of $\mathrm{DM}_{\mathrm{B}}$ is known. At each current time, $n$ new DMs are calculated and the mean and variance are determined as before so that the probability density function of $\mathrm{DM}_{\mathrm{C}}$ is now known. The basis for this method is then to compare the distribution of a current DM to the baseline DM to determine if there is a change in the underlying distribution, thus indicating a potential change in the structure and possible damage.

3.1. Statistical Test. In previous analytical work, a normal difference method was used to compare the distribution of $\mathrm{DM}_{\mathrm{C}}$ to the $\mathrm{DM}_{\mathrm{B}}[26]$. This was appropriate because the number of samples in each distribution was set equal to each other. For the actual implementation, the sample sizes of each distribution are likely to be different due to the actual varying truck volumes over specific time periods. It is also assumed that the variance of the two samples is unequal. With these considerations, Welch's $t$-test is used to compare distributions [27]. Welch's $t$-test defines the statistic $t$ as:

$$
t=\frac{\overline{X_{H}}-\overline{X_{C}}}{s_{\overline{X_{H}}-\overline{X_{C}}}},
$$

where

$$
s_{\overline{X_{H}}-\overline{X_{C}}}=\sqrt{\frac{s_{H}^{2}}{n_{H}}+\frac{s_{C}^{2}}{n_{C}}}
$$

$\overline{X_{H}}$ and $\overline{X_{C}}, s_{H}^{2}$ and $s_{C}^{2}$, and $n_{H}$ and $n_{C}$ are the mean, variance, and sample size, respectively, of the healthy and current distributions. The degrees of freedom $v$ is approximated using the Welch-Satterthwaite equation:

$$
v=\frac{\left(\left(s_{H}^{2} / n_{H}\right)+\left(s_{C}^{2} / n_{C}\right)\right)^{2}}{\left(s_{H}^{4} / N_{H}^{2}\left(N_{H}-1\right)\right)+\left(s_{C}^{4} / N_{c}^{2}\left(N_{c}-1\right)\right)} .
$$

The $t$-distribution can be used to test the null hypothesis that the two DM means are equal at a certain significance level. If the current $t$-statistic is smaller in absolute value than the critical $t$-value for a particular level of significance, the null hypothesis is rejected; this indicates a statistically significant change in the mean and potential for damage in the structure. If the current $t$-statistic is greater in absolute value than the critical $t$-value for a particular level of significance, the null hypothesis cannot be rejected; this indicates no 
statistically significant change in the mean and that the structural integrity of the current scenario should be assumed the same as the structural integrity of the baseline scenario.

To provide further insight into the statistical test the $P$ values instead of a general "pass" or "fail" result are observed. The $P$ value is the probability of observing an event at least as extreme as the one actually observed, given that the null hypothesis is true. The $P$ value for a two-tailed Welch's $t$-test can be calculated as:

$$
P=2[1-\Phi(t)] .
$$

Generally one rejects the null hypothesis when the $P$ value is smaller than or equal to the significance level.

3.2. Thermal Effects. To account for thermal effects on the damage measures, each DM is paired with an associated temperature. Since tilt and temperature data are collected simultaneously, this data is already paired. The acceleration data is paired with the temperature record that has a timestamp closest to that of the acceleration data. The paired data sets are distributed into temperature bins. For tilt data, the temperature bins have a range of $0.056^{\circ} \mathrm{C}\left(0.1^{\circ} \mathrm{F}\right)$ and for the acceleration data the temperature bins have a range of $0.56^{\circ} \mathrm{C}\left(1.0^{\circ} \mathrm{F}\right)$. A smaller bin is used for tilt data because it is more dependent on temperature than the acceleration-based DMs. When the probabilistic SHM method is applied the distribution of the DM from each temperature bin of the baseline year is compared to that of the same temperature bin for the current year. A temperature dependant $P$ value, $P_{T_{1}<T<T_{2}}$, is determined. The associated temperature dependant $P$ values are averaged over all of the temperature bins such that:

$$
\bar{P}=\frac{1}{N} \sum_{n=1}^{N} p_{\Delta T \cdot(n-1)<T<\Delta T \cdot n}
$$

where $\bar{P}$ is the average $P$ value for all temperatures, $N$ is the number of temperature bins, and $\Delta T$ is the size of the temperature bin. The $\bar{P}$ value is then compared to a prescribed significance level to determine if the null hypothesis, that the means are equal, can be rejected.

\section{Verification of the Probabilistic SHM Method Using Historical Data}

To verify the proposed method, actual bridge data is used. The baseline data from September 2001-August 2002, when monitoring of this bridge began, is compared to the most recent 10-month data set, from September 2003-August 2004. It should be noted that during each of these years data was only collected 10 months out of each year due to ongoing system maintenance. While bridge inspections over this threeyear time period verify that there is no structural damage to the bridge, the bridge did experience a permanent rotation between September 2002 and August 2003. Additionally, an accelerometer sensor was observed to not function properly during this period. Both events provided the opportunity for verifying the ability of the SHM system to detect a change in
Tilt data versus time from September 2001-August 2004

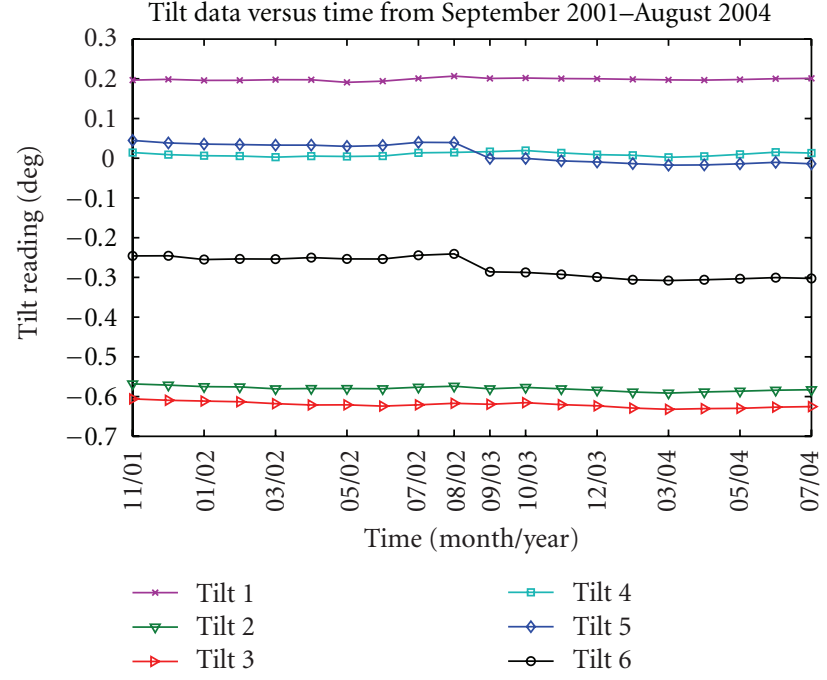

Figure 5: Tilt data versus time from September 2001 to August 2004 for Hartford Flyover Bridge.

the structural behavior of the system. This study considers the verification of the SHM method using actual measured data that happens to include a permanent tilt in the bridge structure. While further analytical studies including a high fidelity finite element model and a suite of likely damage scenarios would be necessary to verify the reliability and robustness of the proposed probabilistic approach for other damage conditions, the verification provided in this study is simply intended to illustrate, using actual data available on the bridge, the ability of the method to identify a major change in the structure.

In a previous study [22], the Hartford Flyover Bridge was observed to have undergone a structural change noted as a permanent rotation in two of the tiltmeters occurring between September 2002 and August 2003. This historical data was manually saved prior to and after this period. A plot of average monthly tilt versus time for all tilt sensors is shown in Figure 5. From this figure it can be seen that over the course of a year the average monthly tilt measurements vary with temperature between the seasons however between September 2002 and August 2003 a permanent rotation occurred in tiltmeters 5 and 6 that never recovered. Since there was no other observable permanent rotation in tiltmeters it is postulated that the movement in meters 5 and 6 is due to a movement in the structure and not drift in the sensor [22]. In this section the three DMs are identified and $P$ values determined comparing the baseline and current DM distributions.

4.1. Natural Frequency Damage Measure. The premise of checking the natural frequency is that a change in the stiffness of the structure, presumably due to structural damage, will result in a change in the natural frequency. Checking the first, or fundamental, natural frequency will provide a global measure as to the structural health of the structure. To estimate the fundamental natural frequency of the bridge the power spectral density functions of the eight acceleration 
TABLe 1: $P$ values for comparison of natural frequency distributions.

\begin{tabular}{ccccccccc}
\hline & AV1 & AV2 & AH3 & AV4 & AV5 & AH6 & AV7 & AV8 \\
\hline$P$ values & 0.4098 & 0.3904 & 0.3863 & 0.4019 & 0.3695 & 0.2531 & 0.3312 & 0.3262 \\
\hline
\end{tabular}

TABle 2: $P$ values for comparison of peak acceleration distributions.

\begin{tabular}{cccccccccc}
\hline & AV1 & AV2 & AH3 & AV4 & AV5 & AH6 & AV7 & AV8 \\
\hline$P$ values & 0.3734 & 0.3971 & 0.2392 & 0.3680 & 0.3916 & 0.0267 & 0.4328 & 0.4398 \\
\hline
\end{tabular}

measurements are determined, where $G_{y y}(f)$ is the onesided autospectra of the measured absolute acceleration [28]. The spectral density functions are defined as:

$$
G_{y y}(f)=2 \lim _{T \rightarrow \infty} \frac{1}{T} E\left[\left|Y_{k}(f, T)\right|^{2}\right],
$$

where $Y_{k}(f, T)$ is calculated using the finite Fourier transform (FFT) of the measured output, $f$ is frequency, $T$ is the record length, and $E[\cdot]$ is the expected value operator over the ensamble index $k$ in question. An FFT is performed on each record of collected acceleration data. Using these functions, the first natural frequency of the bridge can be estimated as the corresponding frequency, $f$, of the maximum value of the real-valued autospectral density function $G_{y y}(f)$ while searching over a range of $\pm 10 \%$ of the measured baseline natural frequency.

The natural frequencies calculated during September 2001 to August 2002 represent the baseline scenario. The natural frequencies calculated during September 2003 to August 2004 represent the current scenario. Table 1 shows the $P$ values as calculated from (5) for each accelerometer collecting data when Welch's $t$-test is applied at 5\% significance with a null hypothesis that the mean natural frequency from each year is the same.

All $P$ values exceed 5\% (0.05) significance so the observation is consistent with the null hypothesis - that the means of the natural frequencies are the same for the current period as for the baseline period, and thus the structure is healthy.

4.2. Peak Acceleration Damage Measure. The peak acceleration provides a local measure of the response and is expected to change in the presence of structural damage. This time domain measurement is able to provide meaningful results even in the presence of potential nonlinear behavior. The peak acceleration was calculated for each triggered truck crossing during September 2001 to August 2002 to represent the baseline scenario. The peak accelerations calculated during September 2003 to August 2004 represent the current scenario. Table 2 shows the $P$ values as calculated from (5) for each accelerometer collecting data when Welch's $t$-test is apply at 5\% significance with a null hypothesis that the mean peak acceleration from each year is the same.

Table 2 shows that with exception to AH6, the null hypothesis cannot be rejected and it is likely that the structure is still healthy. Sensor AH6 is observed during the current period to have a permanent offset. The sensor was removed and recalibrated to remove the offset. Thus the SHM method identified here a change in the sensor, not a change in the
TAble 3: $P$ values for comparison of tilt distributions.

\begin{tabular}{ccccccc}
\hline & Tilt 1 & Tilt 2 & Tilt 3 & Tilt 4 & Tilt 5 & Tilt 6 \\
\hline$P$ values & 0.1491 & 0.0084 & 0.0431 & 0.3096 & 0.0000 & 0.0000 \\
\hline
\end{tabular}

structural system. This example illustrates how any SHM method should not be considered to replace regular bridge inspections but should supplement these inspections and indicate when there is a significant statistical event to warrant further investigation.

4.3. Tilt Damage Measure. As Virkler [25] pointed out, the original purpose of using tiltmeters on the Hartford Flyover Bridge was to see if excessive tilting of the superstructure was the cause of the noticed column cracking. Thus, five of the tiltmeters were placed at piers and one at the midspan of Span 4. Olund [22] showed that during the winter of 20022003 there was a recorded permanent tilt of the bridge. The proposed probabilistic SHM method is applied to the tilt data. Applying Welch's $t$-test at 5\% significance to the tilt distributions for the distributions of the baseline and current tilt DMs gives the following $P$ values in Table 3 .

Table 3 shows the $P$ values for tiltmeters 5 and 6 are zero which verifies Olund's [22] conclusion. Additionally, the $P$ values for tiltmeters 2 and 3 indicate there may have been more extensive, yet slight, tilt occurring over the length of the bridge whereby Span 4 in addition to the previously identified Span 6 may have undergone permanent drift. This result shows that using a probabilistic SHM method can show statistical significance in the difference of two values when a person physically observing the data might not be able to see this change.

\section{Field Implementation of the SHM Method}

The verification of the probabilistic SHM method was verified using actual measured data during a period where permanent tilt in the bridge was observed. The data used in this verification is from 2001-2004 was collected by a bridge monitoring system that automatically triggered and saved raw data and required the user to manually archive and process this data. A viable long term implementation of an SHM method on an actual highway bridge will require less manual effort. A fully automated system is being deployed on the Hartford Flyover Bridge that will trigger, process, save locally, and regularly archive data. The proposed SHM method is implemented at the bridge on a PC located in a cabinet 


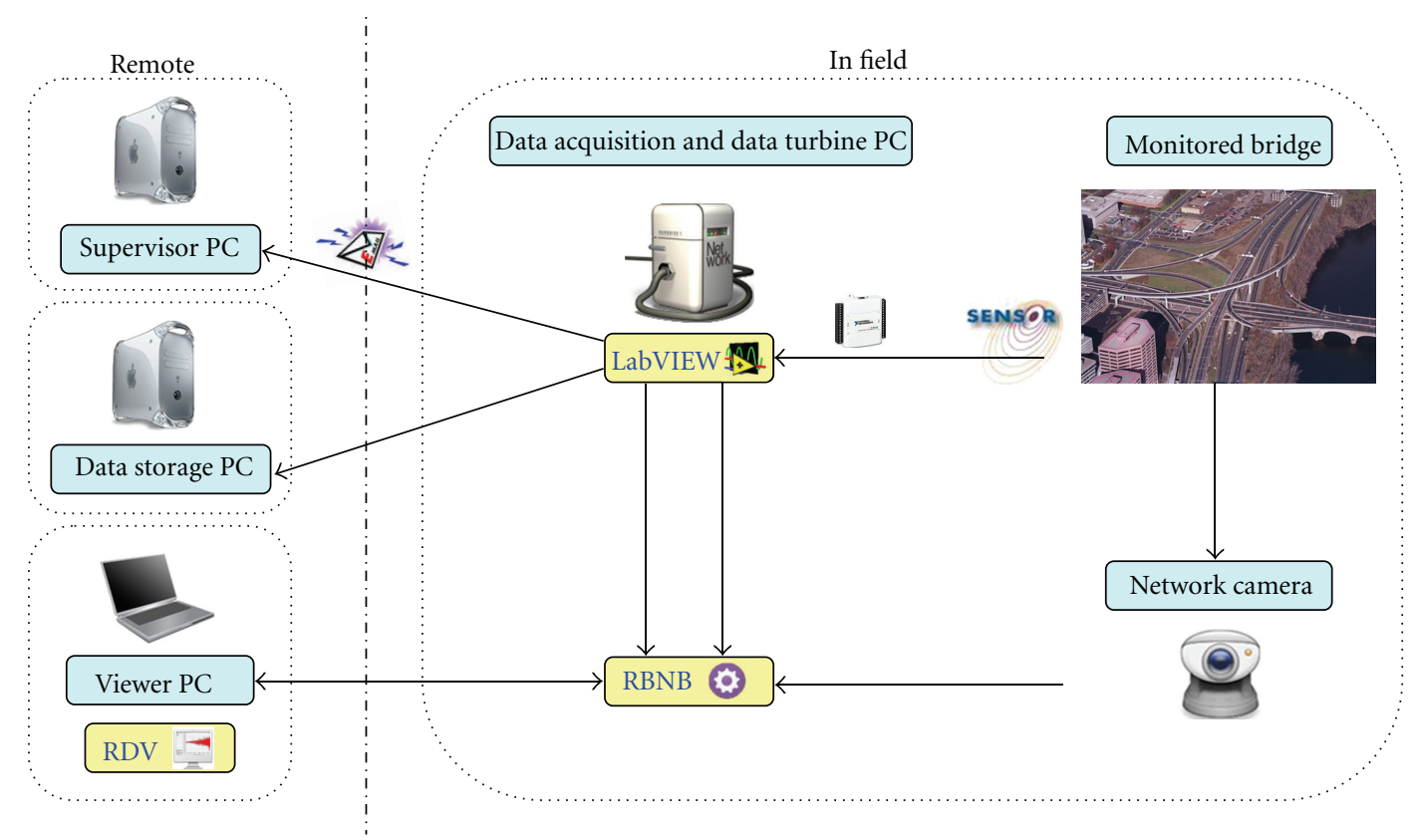

FIGURE 6: Automated bridge monitoring system architecture.

under the bridge with a Microsoft Windows Platform, using LabVIEW Software and National Instruments hardware for data acquisition. The system components and architecture are based on those proposed by Jiang [29]. A schematic of the system architecture can be seen in Figure 6.

Sensor measurements are collected by the National Instruments data acquisition system connected to the onsite PC. Once sampled and digitized, the measurements are processed in LabVIEW and data is collected and archived for later retrieval for SHM. In addition to automated processing and archiving to an FTP site, real-time viewing of streaming data and video is available.

Data acquisition refers to the sampling and analog to digital (A/D) conversion of the sensor measurements. The data acquisition system is a 32-channel analog input, 16-bit, 1.25 MS/s single-channel system, compatible with LabVIEW. Acceleration, tilt, and temperature measurements are acquired at different intervals and sampling rates. The tilt and temperature data are continuously collected at a sampling rate of 10 minutes (a single measurement is taken once every 10 minutes). Acceleration data is acquired by the system when triggered by an acceleration measurement of AV2 exceeding the predefined threshold of $0.0095 \mathrm{~g}$. This trigger level corresponds to the crossing of a truck, which ensures sufficient excitation of the bridge. Once triggered, a record is saved with a sampling frequency of $100 \mathrm{~Hz}$, starting $14 \mathrm{sec}$ onds prior to the triggering event, and lasting for a duration of 30 seconds.

The digitized data is processed in LabVIEW according to the probabilistic SHM method presented in this paper. If an anomaly in the data occurs during the SHM method, an email is generated stating the anomaly in the bridge behavior which is sent to another remote PC for a supervisor to investigate. Both raw and processed data are sent via FTP automatically to a remote PC at the Connecticut Department of Transportation for data storage.

Data is also streamed from the onsite PC to a data turbine program in real-time. Data Turbine is a high performance time-synchronized data streaming service [30]. Data Turbine allows data captured from a network video camera and the data acquisition system to be synchronized and stored in a buffer for viewing. This allows remote users to view the data and video in either real-time or in playback mode through the buffered data in Data Turbine. Data Viewing is accomplished through a Java-based software called the Real-Time Data Viewer (RDV) which was originally developed by The Network for Earthquake Engineering Cyberinfrastructure Center (NEESit) now NEEShub (http://nees.org/).

\section{Conclusion}

This paper uses an in-service highway bridge to verify a probabilistic SHM method. The bridge selected for this study is a curved steel box-girder with composite concrete deck located in Hartford, CT, USA. The bridge is being monitored as part of the University of Connecticut's long-term bridge monitoring project. The bridge is outfitted with accelerometers, temperature sensors, and tiltmeters. The damage measures studied are the fundamental natural frequency, peak accelerations, and tilt. The probabilistic SHM method uses Welch's t-test to compare damage measure distributions. The approach verifies the permanent rotation first observed by Olund [22] and that there is no structural damaged as observed by regular bridge inspections. It has also been shown through the error in accelerometer AH6 that this probabilistic BHM method is meant to complement regular 
bridge inspections and not to replace them. An automated bridge monitoring system is described with the probabilistic structural health monitoring method implemented on an actual highway bridge.

\section{Acknowledgments}

University of Connecticut, the Connecticut Transportation Institute, and the Connecticut Department of Transportation are acknowledged for their joint participation in the ongoing bridge monitoring project funded by the Connecticut Department of Transportation and the Federal Highway Administration. This paper, prepared in cooperation with the Connecticut Department of Transportation and the Federal Highway Administration, does not constitute a standard, specification, or regulation. The contents of this paper reflect the views of the authors who are responsible for the facts and the accuracy of the data presented herein. The contents do not necessarily reflect the views of the Connecticut Department of Transportation or the Federal Highway Administration.

\section{References}

[1] S. W. Doebling, C. R. Farrar, M. B. Prime, and D. W. Shevitz, "Damage identification and health monitoring of structural and mechanical systems from changes in their vibration characteristics: a literature review," Report LA-I3070-MS, Los Alamos National Laboratory, Los Alamos, NM, USA, 1996.

[2] S. W. Doebling, F. M. Hemez, L. D. Peterson, and C. Farhat, "Improved damage location accuracy using strain energybased mode selection criteria," AIAA Journal, vol. 35, no. 4, pp. 693-699, 1997.

[3] S. W. Doebling, C. R. Farrar, M. B. Prime, and D. W. Shevitz, "A review of damage identification methods that examine changes in dynamic properties," Shock and Vibration Digest, vol. 30, no. 2, pp. 91-105, 1998.

[4] F. K. Chang, Structural Health Monitoring: Current Status and Perspectives, Technomic, Lancaster, Pa, USA, 1997.

[5] S. B. Chase and G. Washer, "Nondestructive evaluation for bridge management in the next century," Public Roads, vol. 61, no. 1, pp. 16-25, 1997.

[6] L. D. Olson and C. C. Wright, "Nondestructive testing for repair and rehabilitation," Concrete International, vol. 12, no. 3, pp. 58-64, 1990.

[7] C. R. Farrar, W. B. Baker, T. M. Bell et al., "Dynamic characterization and damage detection in the I-40 bridge over the Rio Grande," Report LA-12767-MS UC-906, Los Alamos National Laboratory, Los Alamos, NM, USA, June 1994.

[8] A. Chakraborty and D. Okaya, "Frequency-time decomposition of seismic data using wavelet-based methods," Geophysics, vol. 60, no. 6, pp. 1906-1916, 1995.

[9] O. S. Salawu and C. Williams, "Bridge assessment using forced-vibration testing," Journal of Structural Engineering, vol. 121, no. 2, pp. 161-173, 1995.

[10] C. R. Farrar and S. W. Doebling, "Lessons learned from applications of vibration based damage identification methods to large bridge structure," in Structural Health Monitoring: Current Status and Perspectives, F. K. Chang, Ed., pp. 351-370, Technomic Publishing, Lancaster, Pa, USA, 1997.
[11] O. S. Salawu, "Detection of structural damage through changes in frequency: a review," Engineering Structures, vol. 19, no. 9, pp. 718-723, 1997.

[12] C. R. Farrar, S. W. Doebling, and D. A. Nix, "Vibration-based structural damage identification," Philosophical Transactions of the Royal Society A, vol. 359, no. 1778, pp. 131-149, 2001.

[13] J. Caicedo, S. J. Dyke, and E. A. Johnson, "Health monitoring based on component transfer functions," in Advances in Structural Dynamics, J. M. Ko and Y. L. Xu, Eds., vol. 2, pp. 9971004, 2000.

[14] F. K. Chang, Structural Health Monitoring 2000, Technomic, Lancaster, Pa, USA, 2000.

[15] H. Sohn, Charles R. Farrar, Michael L. Fugate, and Jerry J. Czarnecki, "Structural health monitoring of welded connections," in Proceedings of the 1st International Conference on Steel \& Composite Structures, Pusan, Korea, June 2001.

[16] H. Sohn, M. Dzwonczyk, E. G. Straser, A. S. Kiremidjian, K. H. Law, and T. Meng, "An experimental study of temperature effect on modal parameters of the Alamosa Canyon Bridge," Earthquake Engineering and Structural Dynamics, vol. 28, no. 7-8, pp. 879-897, 1999.

[17] J. T. Dewolf, M. P. Culmo, and R. G. Lauzon, "Connecticut's bridge infrastructure monitoring program for assessment," Journal of Infrastructure Systems, vol. 4, no. 2, pp. 86-90, 1998.

[18] DeWolf and T. John, "Structural health monitoring of three bridges in connecticut," in Proceedings of the 88th Annual Meeting Transportation Research Board Annual Meeting (TRB '09), 2009.

[19] R. G. Lauzon and J. T. DeWolf, "Connecticut's bridge monitoring program: making important connections last," TR News, no. 224, p. 46, 2003.

[20] J. Olund and J. DeWolf, "Passive structural health monitoring of Connecticut's bridge infrastructure," Journal of Infrastructure Systems, vol. 13, no. 4, pp. 330-339, 2007.

[21] C. Liu and J. T. DeWolf, "Effect of temperature on modal variability of a curved concrete bridge under ambient loads," Journal of Structural Engineering, vol. 133, no. 12, pp. 1742-1751, 2007.

[22] J. Olund, Long term structural health monitoring of Connecticut's bridge infrastructure with a focus on a composite steel tub-girder bridge, M.S. thesis, The University of Connecticut, Storrs, Conn, USA, 2006.

[23] A. Rytter, Vibration based inspection of civil engineering structures, Ph.D. thesis, Aalborg University, Aalborg, Denmark, 1993.

[24] Q. W. Zhang, "Statistical damage identification for bridges using ambient vibration data," Computers and Structures, vol. 85, no. 7-8, pp. 476-485, 2007.

[25] C. Virkler, Continuous structural monitoring coupled with finite element modeling for a composite steel box girder building, M.S. thesis, The University of Connecticut, Storrs, Conn, USA, 2005.

[26] A. M. Scianna and R. Christenson, "Probabilistic structural health monitoring method applied to the bridge health monitoring benchmark problem," Transportation Research Record, no. 2131, pp. 92-97, 2009.

[27] A. H.-S. Ang and W. H. Tang, Probability Concepts in Engineering Planning and Design, Basic Principles, vol. 1, John Wiley \& Sons, New York, NY, USA, 1975.

[28] J. S. Bendat and A. G. Piersol, Random Data Analysis and Measurement Procedures, Probability and Statistics, John Wiley \& Sons, New York, NY, USA, 3rd edition, 2000. 
[29] Z. Jiang, The use of NEES cyberinfrastructure in structural health monitoring and earthquake education, M.S. thesis, The University of Connecticut, Storrs, Conn, USA, 2008.

[30] DataTurbine: http://www.dataturbine.org/. 

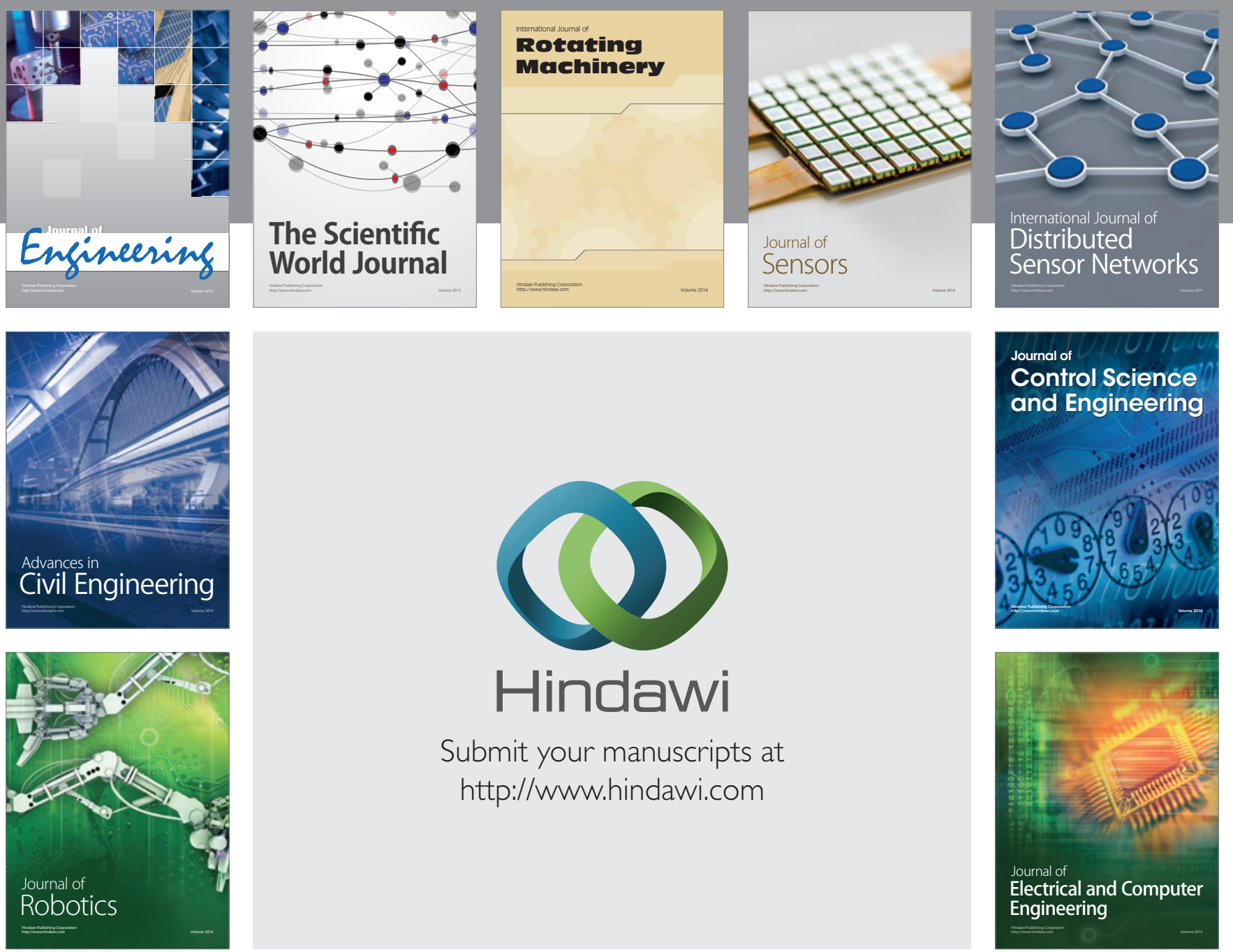

Submit your manuscripts at

http://www.hindawi.com
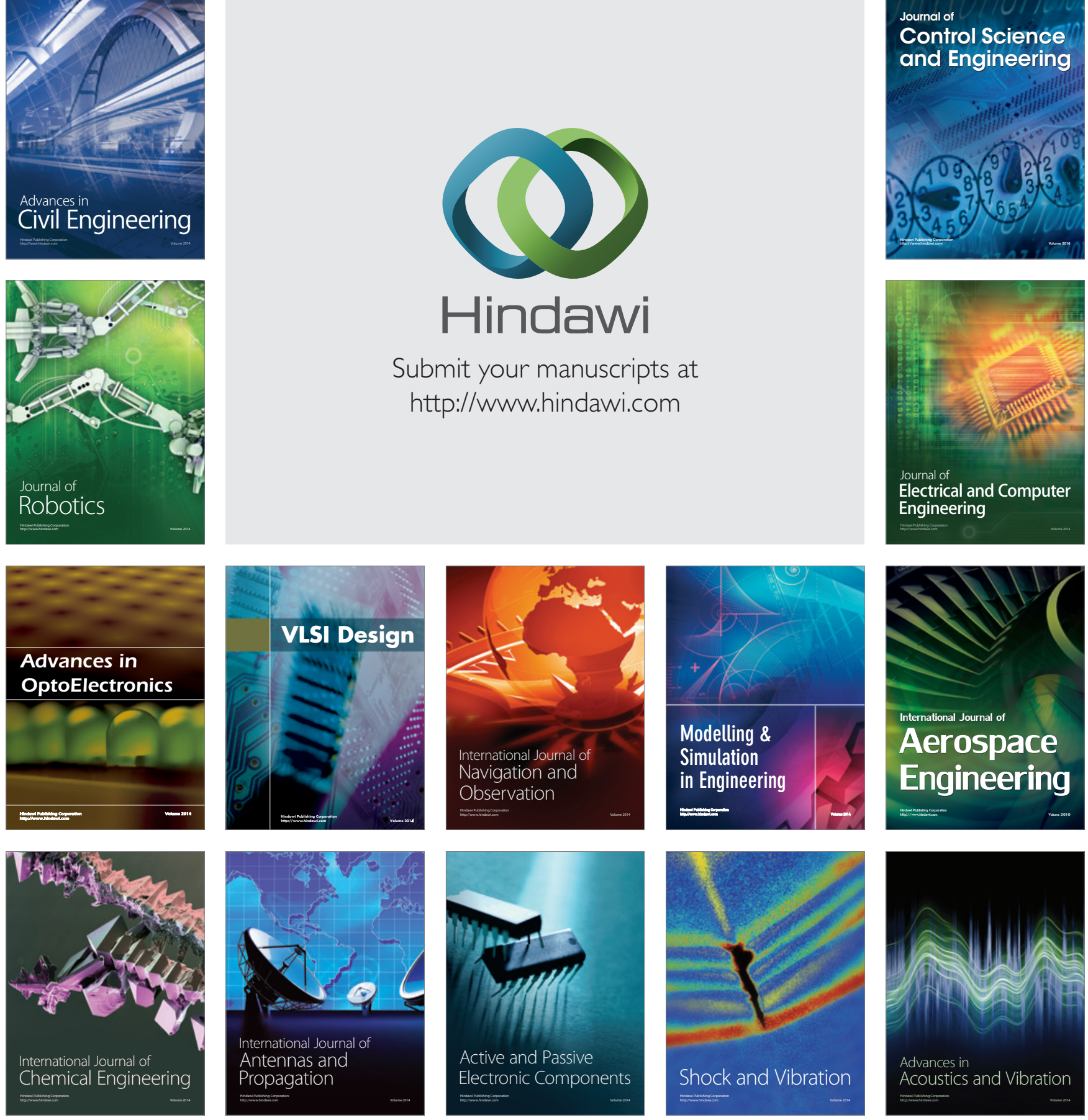